\title{
Genome survey sequencing of common vetch (Vicia sativa $L$.) and genetic diversity analysis of Chinese germplasm with genomic SSR markers
}

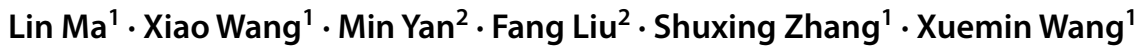

Received: 20 August 2021 / Accepted: 22 October 2021 / Published online: 6 November 2021

(c) The Author(s) 2021

\begin{abstract}
Background Common vetch (Vicia sativa L.) is an annual legume with excellent suitability in cold and dry regions. Despite its great applied potential, the genomic information regarding common vetch currently remains unavailable.

Methods and results In the present study, the whole genome survey of common vetch was performed using the next-generation sequencing (NGS). A total of $79.84 \mathrm{Gbp}$ high quality sequence data were obtained and assembled into 3,754,145 scaffolds with an N50 length of $3556 \mathrm{bp}$. According to the K-mer analyses, the genome size, heterozygosity rate and GC content of common vetch genome were estimated to be $1568 \mathrm{Mbp}, 0.4345$ and 35\%, respectively. In addition, a total of 76,810 putative simple sequence repeats (SSRs) were identified. Among them, dinucleotide was the most abundant SSR type (44.94\%), followed by Tri- (35.82\%), Tetra- (13.22\%), Penta- (4.47\%) and Hexanucleotide (1.54\%). Furthermore, a total of 58,175 SSR primer pairs were designed and ten of them were validated in Chinese common vetch. Further analysis showed that Chinese common vetch harbored high genetic diversity and could be clustered into two main subgroups.

Conclusion This is the first report about the genome features of common vetch, and the information will help to design whole genome sequencing strategies. The newly identified SSRs in this study provide basic molecular markers for germplasm characterization, genetic diversity and QTL mapping studies for common vetch.
\end{abstract}

Keywords Chinese common vetch $\cdot$ Genome survey $\cdot$ SSR $\cdot$ Genetic diversity

\section{Introduction}

Common vetch (Vicia sativa $\mathrm{L}$.) is one kind of self-pollinating annual legume with excellent suitability in cold and dry regions, such as western Asia and northern Africa [1]. As an inexpensive and rich source of protein, common vetch grows quickly and is normally used for feeding livestock on account of its high digestibility [2]. In addition, common vetch can also be used as a green manure crop on idle farmland due to its strong ability to fix nitrogen [3]. Moreover, following with the increasing global population and plant-based

Lin Ma and Xiao Wang have contributed equally to this work.

Xuemin Wang

wangxuemin@caas.cn

1 Institute of Animal Sciences, Chinese Academy of Agricultural Sciences, Beijing 100193, China

2 National Animal Husbandry Station, Ministry of Agriculture Sciences, Beijing 100125, China protein demands, common vetch could be exploited as a future potential protein source due to its widely adaption in marginal cropping zones with severe drought and cold conditions [4].

However, the genetic information of common vetch remains largely unknown, although some studies have performed transcriptome analysis, genetic diversity analysis with ESTs based on SSR markers, and EMS-induced mutation development [2, 5-7]. Nevertheless, the genomic information regarding common vetch currently remains unavailable, as well as the systematic analysis of Chinese common vetch germplasm. The lack of the reference genome sequence impedes the advances in functional genomics and molecular breeding of this species [8]. Therefore, it is necessary to conduct the genome survey sequencing which would obtain basic knowledge on the genome structure of common vetch, providing a foundation for the further research of this specie.

Recently, the next-generation sequencing (NGS) has been employed as the cost-effective approach to conduct genome 
survey sequencing [9-11]. Except the basic knowledge of genome structure, the genome survey sequencing will provide a large number of simple sequence repeats (SSRs), which could be developed into molecular markers [12-14]. As the versatile DNA-based markers one kind of markers, SSRs markers showed multiple advantages including codominant, more informative and more economical, which were generally used in plant genetic researches, such as population diversity, genetic linkage mapping and evolutionary studies $[15,16]$. A larger number of SSR markers are essential for comprehensive genome-wide association studies (GWAS), as well as the quantitative trait locus mapping (QTL) and marker-assisted selection (MAS) [14, 17].

In the present research, we perform the de novo whole genome sequencing of common vetch through NGS, and then assembly to construct a reference genome database. The results showed that the genome of common vetch was estimated to be $1568 \mathrm{Mbp}$ with a heterozygosity of $0.4345 \%$. A total of 76,810 putative SSRs were identified and 58,175 of them were designed as potential SSR markers. In addition, 10 SSRs were validated in 68 Chinese common vetch accessions and suggested the high genetic diversity of Chinese common vetch. Taken together, this study firstly reported the de novo whole genome sequencing of common vetch and firstly analyzed the genetic diversity of Chinese germplasm resources. The genome database and potential SSR markers would provide the foundation for further genomic functional and evolutionary analyses of common vetch, as well as accumulating the development of its molecular breeding.

\section{Materials and methods}

\section{Plant materials and growth condition}

The common vetch (Vicia sativa) cv. 'Lanjian No.1' from Lanzhou University (Lanzhou, Gansu, China) was chosen for the genome survey. The seeds were planted in $10 \mathrm{~cm}$ pots and grown in the greenhouse at $24 / 22{ }^{\circ} \mathrm{C}$ (day/night) temperature with $16 \mathrm{~h}$ light (380-400 $\left.\mu \mathrm{E} / \mathrm{m}^{2} / \mathrm{s}\right)$. After growing for three weeks, fresh leaves from one individual plant were collected and quickly frozen in liquid nitrogen for DNA isolation.

A total of 68 common vetch accessions originating from China were used for genetic diversity analysis (Table S1). In addition, 20 common vetch accessions originating from worldwide were used for SSR polymorphism selection (Table S2). The seeds were sterilized in $75 \%$ ethanol for $5 \mathrm{~min}$ and rinsed with sterile water five times. They were placed on filter paper in dishes and then subsequently cultured in a growth chamber at $25{ }^{\circ} \mathrm{C}$. The eight-day-old seedlings were prepared for DNA extraction.

\section{DNA extraction and genome sequencing}

Total genomic DNA was isolated by using the CTAB method with modifications [18]. DNA concentrations were measured on a Nanodrop (Thermo Fisher Scientific, Waltham, MA). DNA quality was detected on a Qubit (Thermo Fisher Scientific, Waltham, MA). The genomic paired-end library with 300-400 bp short-inserts was constructed and sequenced on an Illumina NovaSeq 6000 (Illumina Inc. San Diego, CA, USA) with PE 150 sequencing methods.

\section{K-mer analyses and genome size estimation}

All of the clean data were used for K-mer analysis using Jellyfish software [19]. Based on the results of K-mer frequency distributions (K-mer $=17$ ), the characteristics of the genome, including genome size, repeat content and heterozygosity rate, were estimated by using GenomeScope [20].

\section{Genome assembling and guanine plus cytosine (GC) content analysis}

SOAPdenovo software was used for genome assembly [21]. In brief, a de Bruijn graph was constructed based on the overlapping relationship reads from SOAPdenovo software, and contigs were output after simplifying the de Bruijn graph. Scaffolds were constructed based on the contigs, and gaps inside the scaffolds were filled by employing GAPCloser. Here, $10-\mathrm{kb}$ nonoverlapping sliding windows along the assembled sequence were hired to calculate the average GC sequencing depth [22].

\section{Genomic microsatellite identification}

The MIcroSAtellite (MISA) software (http://pgrc.ipk-gater sleben.de/misa/misa.html) was employed to detect the genomic SSRs. The search parameters were respectively set for identifying various types of SSRs, including Di-, Tri-, Tetra-, Penta- and Hexa-nucleotide SSR motifs with a minimum of 6, 5, 4, 4 and 4 repeats, respectively. Primer 3 software were used for primers designing for each SSR locus, with the following parameters: $18-25$ primer size, 90-250 bp product size, $70 \%$ GC content, and annealing temperature of $55-65{ }^{\circ} \mathrm{C}$.

\section{SSR genotyping}

For SSR polymorphism selection, a total of 20 common vetch accessions were used (Table S2). Firstly, sixty pairs of putative SSR primers were randomly selected to test whether they harbored polymorphisms among 20 common 
vetch varieties with multiply phenotypes by the methods of non-denaturing polyacrylamide gel electrophoresis (PAGE). Then ten SSR were selected for further study.

The selected 10 pairs SSR primers with additional different fluorescent probes on forward primers were used for polymorphism screening of 68 common vetch accessions in China (Table S3). The PCR was conducted in a $20 \mu \mathrm{L}$ reaction system containing $20 \mathrm{ng}$ genomic DNA, $10 \mu \mathrm{L}$ $2 \times$ Taq Master Mix (Genestar, Beijing, China) and $0.5 \mu \mathrm{L}$ each of the forward and reverse primers. The PCR parameters were as follows: $94{ }^{\circ} \mathrm{C}$ for $5 \mathrm{~min} ; 35$ cycles: $94{ }^{\circ} \mathrm{C}$ for $30 \mathrm{~s}, 58^{\circ} \mathrm{C}$ for $30 \mathrm{~s}, 72{ }^{\circ} \mathrm{C}$ for $45 \mathrm{~s}$; the final extension at $72{ }^{\circ} \mathrm{C}$ for $7 \mathrm{~min}$. The PCR products were diluted five times and then resolved in an ABI3130xl Genetic Analyzer (Applied Biosystems, CA, USA). Fragments size and data analysis were determined by using mapmaker 5.0 software.

\section{Genetic diversity analysis}

The allelic diversity and genetic variation parameters including the number of different alleles (Na) and effective alleles $(\mathrm{Ne})$, the index of observed heterozygosity (Ho), expected heterozygosity (He), Shannon's information index (I) and the polymorphism information content (PIC) were calculated by fragment size in GenAlEx 6.5 software [23]. The genetic diversity among the 68 accessions was determined by using dissimilarity analysis and representation for windows (DARwin) software. The dendrogram was generated by using the UPGMA phylogenetic cluster analysis [24].

\section{Results}

\section{Genome sequencing and sequence assembly}

To avoiding the influence the potential heterozygous, we extracted DNA from the single plant leaves of common vetch for libraries constructing (Fig. 1). After filtering the low quality data, we obtained approximately $79.84 \mathrm{Gbp}$ of highquality data from the sequencing library, which were approximately 51 times of the estimated genome size. The Q20 and Q30 of the obtained data were greater than $97 \%$ and $92 \%$, indicating the reliable of the genome survey sequencing. We then de novo assembled $(\mathrm{K}-\mathrm{mer}=75)$ all of the high quality data by using the de Bruijn graph-based SOAPdenovo software. A total of 4,227,942 raw contigs were obtained, and the total length of raw contigs was $1,475,990,986 \mathrm{bp}$ and the contig N50 length of $1245 \mathrm{bp}$ (Table 1). Finally, the assembled common vetch genome consisted of 3,754,145 scaffolds which had a total length of $1,516,858,186 \mathrm{bp}$, and the scaffold N50 length of 3556 bp (Table 1).

\section{Genomic characteristics}

The peak K-mer depth and the number of K-mers were calculated as 45 and 70, 575, 281,718, respectively, based on the K-mer analysis $(\mathrm{K}-\mathrm{mer}=17)$. The genome size of common vetch was estimated at $1568 \mathrm{Mbp}$, while the heterozygosity rate of this genome was $0.4345 \%$, indicating that common vetch was a self-pollinating species (Fig. 2a).

In order to investigate the guanine plus cytosine (GC) content of the common vetch genome, we built a scatterplot
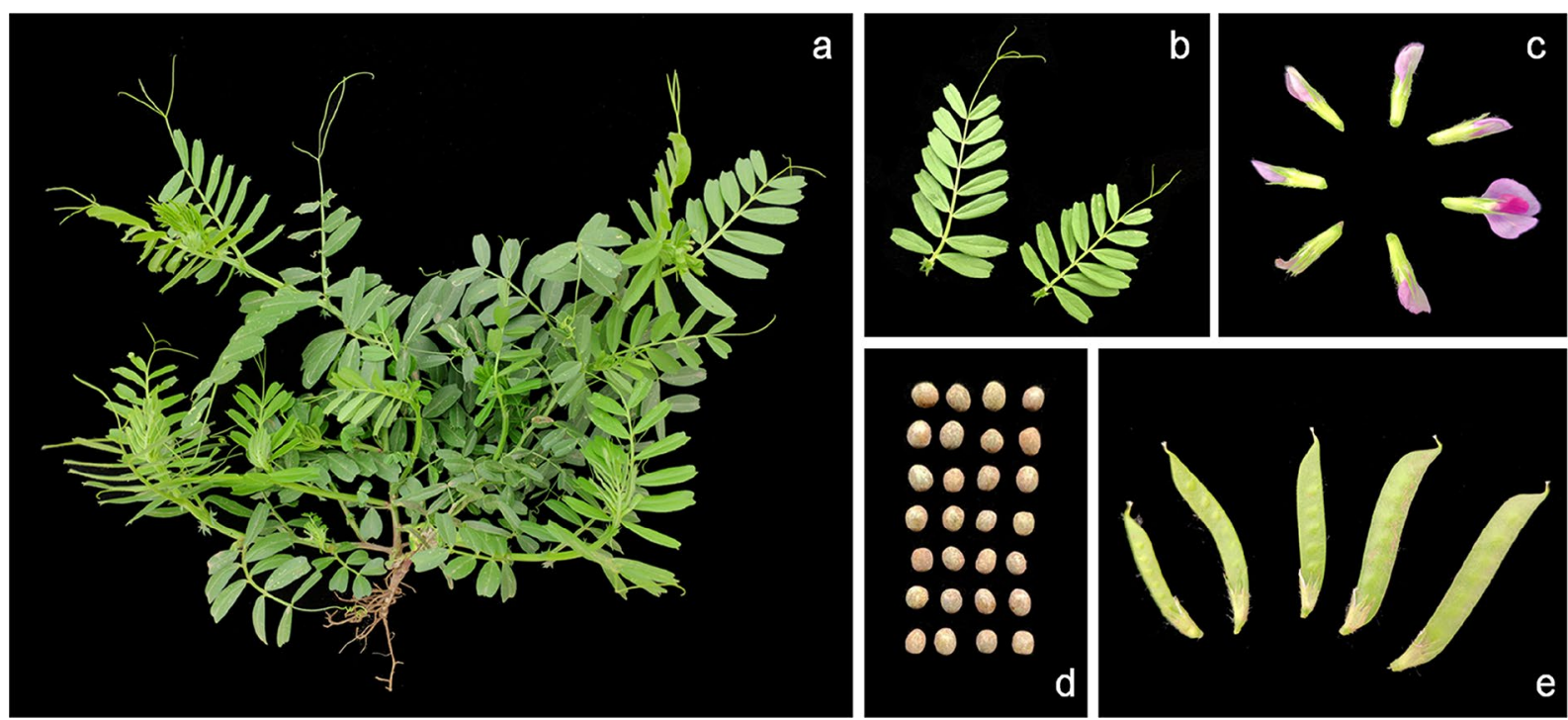

Fig. 1 The morphological characteristics of common vetch cv "Lanjian No.1". a The plant. b The leaves. c The flowers. d The seeds. e The seedpods 
Table 1 Information of the assembled genome sequences of common vetch

\begin{tabular}{ll}
\hline Contigs & \\
\hline Number of sequences & $4,227,942$ \\
Total length (bp) & $1,475,990,986$ \\
Max length (bp) & 38,860 \\
N50 length (bp) & 1245 \\
N90 length (bp) & 115 \\
\hline Scaffolds & \\
\hline Number of sequences & $3,754,145$ \\
Total length (bp) & $1,516,858,186$ \\
Max length (bp) & 89,299 \\
N50 length (bp) & 3556 \\
N90 length (bp) & 116 \\
GC content & $35.94 \%$ \\
\hline
\end{tabular}

graph by using scaffolds larger than $500 \mathrm{bp}$, elucidating the information on sequencing data bias (Fig. 2a). The results showed that the GC content of the common vetch genome was $35 \%$, which was consistent with the main peak in the scatterplot graph. Moreover, we also noticed that the confidence area (shown in red) was around the peak at 35, suggesting that the DNA sample for genome survey sequencing was not polluted by DNA from other species.

\section{Genomic SSR markers development}

The assembled scaffolds were employed for genomic SSR search via the MISA software (http://pgrc.ipk-gatersleben. de/misa/misa.html). A total of 76,810 putative SSRs were identified from 58,373 isoforms and 12,050 isoforms contained more than one SSR. Among the identified putative SSRs, 4932 SSRs were present in compound formation. We found that the most abundant SSR type was Dinucleotide, accounting for $44.94 \%$ of the total SSRs, followed by Tri(35.82\%), Tetra- (13.22\%), Penta- $(4.47 \%)$ and hexa nucleotide (1.54\%) SSRs (Fig. S1). The density of SSRs identified in the assembled common vetch genome was one SSR per $20.41 \mathrm{~kb}$.

The SSRs were categorized by their repeat motifs. The most abundant repeats were AG/CT (17.29\%) and AC/ GT (15.54\%), followed by AT/AT (12.02\%), AAC/GTT (10.07\%), AAT/ATT (9.93\%) and AAG/CTT (9.37\%), and AAAT/ATTT (4.95\%). The most abundant pentanucleotide repeats were AAAAT/ATTTT (1.27\%) and AAACC/CGTTT $(0.79 \%)$ (Fig. S2). Furthermore, we designed primers for 58,175 SSRs by using Primer 3.0 software. The detailed primers are shown in Table S4.

\section{Genetic diversity and cluster analysis of Chinese common vetch}

Ten SSR markers with polymorphisms were selected randomly to investigate genetic diversity of 68 Chinese common vetch accessions. In total, we obtained 76 alleles from the 10 SSR loci (Table 2). For each SSR loci, the number of different alleles $(\mathrm{Na})$ and the effective number of alleles $(\mathrm{Ne})$ were ranged from 3 (SSR-12) to 16 (SSR-13) and 1.2786 (SSR-5) to 6.1286 (SSR-13), respectively. The mean $\mathrm{Na}$ and $\mathrm{Ne}$ were 7.6 and 3.4905. The index of observed heterozygosity (Ho)

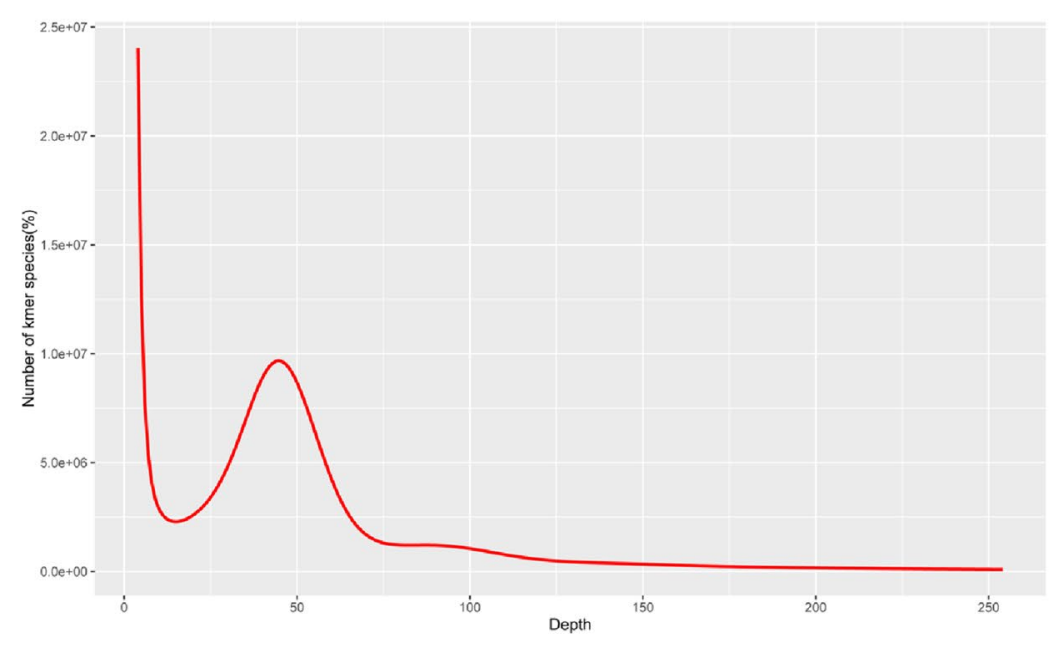

b

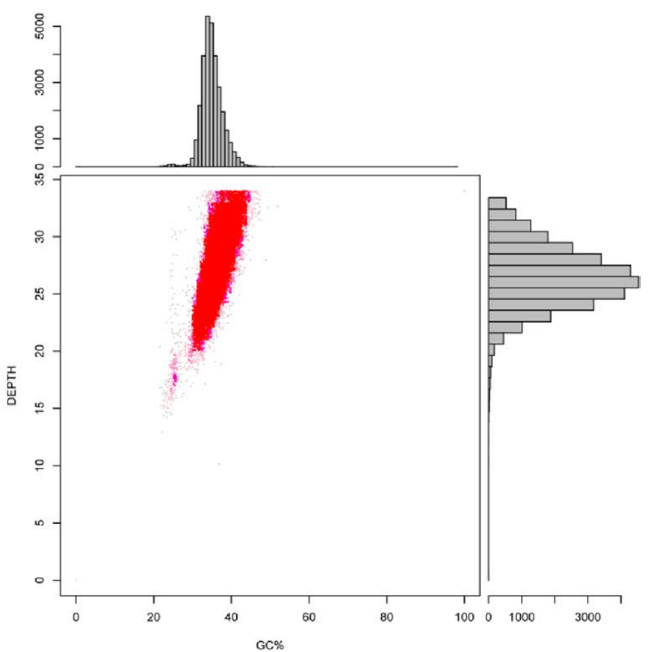

Fig. 2 K-mer frequency distribution at K-mer=17 depth and GC content and depth correction analysis. a The estimated genome size of common vetch was determined based on the following formula: genome size $=\mathrm{K}$-mer depth. The $\mathrm{x}$-axis is depth and $\mathrm{y}$-axis represents the frequency at the particular depth divided by the total frequency of all depths. b The x-axis represents the GC content and the y-axis is the sequence depth. The distribution of the sequence depth is on the right side, while the distribution of the GC content is at the top 
and expected heterozygosity $(\mathrm{He})$ ranged from 0 (SSR-12) to 0.1765 (SSR-10) and 0.3195 (SSR-5) to 0.8430 (SSR13), with the average of 0.0632 and 0.6438 , respectively. The polymorphism information content (PIC) ranged from 0.217802 (SSR-5) to 0.836845 (SSR-13) with an average of 0.639076 . Other parameter, such as Shannon's information index (I), ranged from 0.5341(SSR-5) to 2.1759 (SSR-5) with an average of 1.3387. Together, we noticed that SSR-13 harbored the highest polymorphism, followed by SSR-14, and the polymorphism of SSR-5 was the lowest (Table 2). These results suggested that the 68 common vetch accessions from China harbored high genetic diversity.

In addition, we also constructed the hierarchical tree of the Chinese common vetch accessions based on dissimilarity data, to infer phylogenetic relationships among these 68 accessions. Unweighted neighbor-joining analysis resulted in a dendrogram with two main subgroups (A and B) with 6 and 10 clusters, respectively (Fig. 3). In detail, subgroup A consisted of 33 accessions and most of them were wild accessions or landraces; in contrast, subgroup B was composed of 35 accessions but only 17 of them were wild accessions (4) and landraces (13). In addition, we hardly connected the clusters with their original places, suggesting that more markers should be hired in further population structure analysis.

\section{Discussion}

With the increasing global population, global protein demand is predicted to notably increase by $50 \%$ by 2050 [25]. The extreme climate changes resulting from global warming and the urgent demand for protein have led to the search for suitable species which provide sustainable protein resources [26]. Common vetch (Vicia sativa L.), with excellent drought and cold tolerance, could be exploited to cope with the requirements of humans and livestock [5, 8]. However, the high r-glutamyl-b-cyano-alanine (GBCA) content in common vetch seeds has restricted its application in agriculture, and the traditional strategies have failed to breed common vetch with no GBCA [4].

Modern biotechnology-based high quality references have shown promise in accelerating crop improvement [27]. With the development of functional genomics research, we can obtain the toxin-free common vetch with the use of through modern biotechnology, including overexpression or RNA interference of the candidate genes,

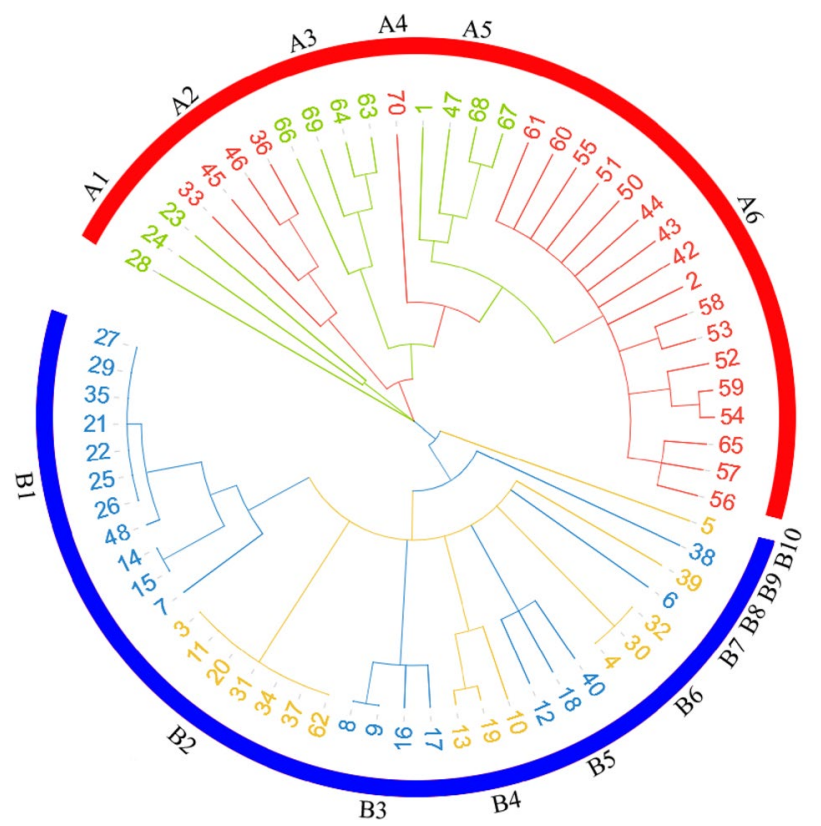

Fig. 3 Cluster diagram for 68 individuals of Chinese common vetch by UPGMA method
Table 2 Diversity statistic from 10 SSR tested in Chinese common vetch accessions $(n=68)$

\begin{tabular}{lllllll}
\hline Locus & $\mathrm{Na}$ & $\mathrm{Ne}$ & $\mathrm{I}$ & Ho & He & PIC \\
\hline SSR-1 & 4 & 3.0552 & 1.1539 & 0.0294 & 0.6777 & 0.672682 \\
SSR-2 & 5 & 2.874 & 1.0305 & 0.0735 & 0.5670 & 0.562873 \\
SSR-3 & 6 & 2.7956 & 1.1717 & 0.0735 & 0.6471 & 0.642264 \\
SSR-5 & 6 & 1.2786 & 0.5341 & 0.0294 & 0.3195 & 0.217802 \\
SSR-9 & 10 & 3.6918 & 1.7656 & 0.0147 & 0.7345 & 0.729144 \\
SSR-10 & 5 & 2.3289 & 0.9759 & 0.1765 & 0.5748 & 0.570627 \\
SSR-11 & 11 & 5.318 & 1.9084 & 0.0441 & 0.8180 & 0.811961 \\
SSR-12 & 3 & 2.0588 & 0.7595 & 0.0000 & 0.5180 & 0.514268 \\
SSR-13 & 16 & 6.1286 & 2.1759 & 0.0735 & 0.8430 & 0.836845 \\
SSR-14 & 10 & 5.9626 & 1.9111 & 0.1176 & 0.8385 & 0.83229 \\
MEAN & 7.6 & 3.4905 & 1.3387 & 0.0632 & 0.6438 & 0.639076 \\
SD & 4.0332 & 1.7276 & 0.5584 & 0.0528 & 0.1897 & 0.188304 \\
\hline
\end{tabular}

$\mathrm{Na}$ observed number of alleles, $\mathrm{Ne}$ effective number of alleles, I Shannon's information index, Ho observed heterozygosity, $\mathrm{He}$ expected heterozygosity 
or editing the genome by CRISPR-Cas (clustered regularly interspaced short palindromic repeats-Cas protein). These biotechnology methods have already been successfully applied in other major crops, such as rice, wheat, maize and barley [28].

In the present study, we performed a genome survey of common vetch through NGS and obtained the genome information, including genome size, heterozygosity and GC content (Table 1, Fig. 1). This approach has been used to analyses a number of plant genomes, such as pistachio [14], Acer truncatum Bunge [29], Akebia trifoliata [30] and yellow horn [31]. The depth distribution $(\mathrm{K}-\mathrm{mer}=17)$ indicated that the genome size of common vetch was 1568 Mbp and the heterozygosity rate was $0.4345 \%$ (Fig. 2). The GC content of the common vetch genome was calculated to be $35 \%$. To our knowledge, this report of genomic information of common vetch is the first of its kind, and lays the foundation for future genome assembly and subsequent functional genome research.

The total number of common vetch populations is quite difficult to estimate, as this specie was distributed worldwide. More than 20,000 accessions were kept in the plant genetic resources (PGR) [2]. It is difficult for farmers to directly use or incorporate into breeding programmes due to the large number of germplasm resources. A core collection for common vetch, which consists of 5\% total accessions and represents $95 \%$ of the genetic diversity, needed to be constructed through evaluating the genetic relationships between accessions [32]. SSRs with significant dominance were used in evaluating genetic diversity in populations, and the genome survey can also provide extremely useful sources for SSR identification [33].

In this study, we identified 76,810 putative SSRs and 58,175 of them were designed as potential SSR markers. Ten of validated SSRs were selected to investigate the genetic diversity of 68 Chinese common vetch accessions with an average PIC was 0.639076 (Table 2). The results showed that the Chinese common vetch accessions harbored high genetic diversity, as well as the efficacy of the SSR markers developed in the present study. The hierarchical tree of 68 Chinese common vetch accessions indicated that these accessions could be clustered into two main subgroups. Further analysis showed that the subgroup A represented the wild and landraces accessions, wherease subgroup B represented the cultivars and commercial variety (Fig. 3). However, 10 SSR markers were largely insufficient for common vetch molecular fingerprint construction and further population structure analysis. Combined with the further research in genome assembly in chromosomal level, the larger number of SSRs identified in this study shows high potential application in construction of the common vetch core collection. Genome-wide association studies (GWAS) in core collection is an effective way for candidate gene identification in functional genome research [2]. Moreover, the larger number of SSR markers are essential for high density linkage map construction in quantitative trait locus (QTL) mapping [34]. The further selection and verification of more SSRs and their corresponding markers should be developed for functional genome research, as well as the molecular marker assisted breeding in the further. Although the study provides the genome features of common vetch, and the information will help to design whole genome sequencing strategies. A further research including chromosomal assembly, gene annotation, SSR mapping, etc. still remains to be analyzed since the complete genomic information is extremely useful.

\section{Conclusion}

In this study, we obtained the first insight into the genome features of common vetch, and the information will help to design whole genome sequencing strategies. The newly identified SSRs were verified in the genetic diversity analysis of Chinese common vetch germplasm resources. This study provides the valuable information for functional genome research in common vetch, as well as the molecular marker assisted breeding in the further.

Supplementary Information The online version contains supplementary material available at https://doi.org/10.1007/s11033-021-06875-z.

Acknowledgements We thank Prof. Yanrong Wang (College of Pastoral Agriculture Science and Technology, Lanzhou University) for gifting the seeds of common vetch cv. 'Lanjian No.1'.

Author contributions LM and XMW conceived and designed the experiments; LM and XW performed the experiment; LM, MY and FL analyzed the data; SXZ cultured plant materials; LM and XMW wrote the manuscript.

Funding This study was supported by the earmarked fund for China Agriculture Research System (CARS-34), National Crop Germplasm Resources Center (NICGR-78) and National Program for Forage Germplasm Conservation (2130135).

Data availability The raw data in this research was deposited in the short read archive (SRA) databank (http://www.ncbi.nlm.nih.gov/sra/) and are available under the accession number PRJNA730328.

\section{Declarations}

Conflict of interests The authors declare no conflict of interest.

Ethical approval The article does not contain any studies with human participants or animals performed by any of the authors.

Consent for publication All the authors have read and consented to submit the manuscript. 
Open Access This article is licensed under a Creative Commons Attribution 4.0 International License, which permits use, sharing, adaptation, distribution and reproduction in any medium or format, as long as you give appropriate credit to the original author(s) and the source, provide a link to the Creative Commons licence, and indicate if changes were made. The images or other third party material in this article are included in the article's Creative Commons licence, unless indicated otherwise in a credit line to the material. If material is not included in the article's Creative Commons licence and your intended use is not permitted by statutory regulation or exceeds the permitted use, you will need to obtain permission directly from the copyright holder. To view a copy of this licence, visit http://creativecommons.org/licenses/by/4.0/.

\section{References}

1. Robertson LD, Singh KB, Erskine W, AbdElMoneim AM (1996) Useful genetic diversity in germplasm collections of food and forage legumes from West Asia and North Africa. Genet Resour Crop Evol 43(5):447-460

2. De la Rosa L, Lopez-Roman MI, Gonzalez JM, Zambrana E, Marcos-Prado T, Ramirez-Parra E (2021) Common Vetch, valuable germplasm for resilient agriculture: genetic characterization and Spanish core collection development. Front Plant Sci 12:1-6

3. Cakmakci S, Aydinoglu B, Karaca M, Bilgen M (2006) Heritability of yield components in common vetch (Vicia sativa L.). Acta Agric Scand B Soil Plant Sci 56:54-59

4. Nguyen V, Riley S, Nagel S, Fisk I, Searle IR (2020) Common vetch: a drought tolerant, high protein neglected leguminous crop with potential as a sustainable food source. Front Plant Sci 11:818

5. Min XY, Liu ZP, Wang YR, Liu WX (2020) Comparative transcriptomic analysis provides insights into the coordinated mechanisms of leaves and roots response to cold stress in common vetch. Ind Crops Prod 158:112949

6. Shi HF, Geng BH, Zhao YL, Liu YJ, Huang RS, Zhao PY, Guo ZF (2020) EMS-induced mutations in common vetch (Vicia sativa L.) and two mutants without anthocyanin accumulation showing increased cold tolerance. Grassl Sci 67(2):148-155

7. Dong R, Dong DK, Luo D, Zhou Q, Chai XT, Zhang JY, Xie WG, Liu WX, Dong Y, Wang YR, Liu ZP (2017) Transcriptome analyses reveal candidate pod shattering-associated genes involved in the pod ventral sutures of common vetch (Vicia sativa L.). Front Plant Sci 8:649

8. De la Rosa L, Zambrana E, Ramirez-Parra E (2020) Molecular bases for drought tolerance in common vetch: designing new molecular breeding tools. BMC Plant Biol 20:71

9. Zhou W, Hu YY, Sui ZH, Fu F, Wang JG, Chang LP, Guo WH, Li BB (2013) Genome survey sequencing and genetic background characterization of Gracilariopsis lemaneiformis (Rhodophyta) based on next-generation sequencing. PLoS ONE 8(7):e69909

10. He Y, Xiao HT, Deng C, Xiong L, Nie H, Peng C (2016) Survey of the genome of Pogostemon cablin provides insights into its evolutionary history and sesquiterpenoid biosynthesis. Sci Rep 6:26405

11. Wang CR, Yan HD, Li J, Zhou SF, Liu T, Zhang XQ, Huang LK (2018) Genome survey sequencing of purple elephant grass (Pennisetum purpureum Schum 'Zise') and identification of its SSR markers. Mol Breed 38:94

12. Jiao Y, Jia HM, Li XW, Chai ML, Jia HJ, Chen Z, Wang GY, Chai CY, van de Weg E, Gao ZS (2012) Development of simple sequence repeat (SSR) markers from a genome survey of Chinese bayberry (Myrica rubra). BMC Genomics 13:201

13. Wei X, Wang LH, Zhang YX, Qi XQ, Wang XL, Ding X, Zhang J, Zhang XR (2014) Development of simple sequence repeat (SSR) markers of Sesame (Sesamum indicum) from a genome survey. Molecules 19(4):5150-5162

14. Motalebipour EZ, Kafkas S, Khodaeiaminjan M, Coban N, Gozel H (2016) Genome survey of pistachio (Pistacia vera L.) by next generation sequencing: development of novel SSR markers and genetic diversity in Pistacia species. BMC Genomics 17:998

15. Varshney RK, Graner A, Sorrells ME (2005) Genetic microsatellite markers in plants: features and applications. Trends Biotechnol 23(1):48-55

16. Zalapa JE, Cuevas H, Zhu HY, Steffan S, Senalik D, Zeldin E, McCown B, Harbut R, Simon P (2012) Using next-generation sequencing approaches to isolate simple sequence repeat (SSR) loci in the plant sciences. Am J Bot 99(2):193-208

17. Blackburn A, Sidhu G, Schillinger WF, Skinner D, Gill K (2021) QTL mapping using GBS and SSR genotyping reveals genomic regions controlling wheat coleoptile length and seedling emergence. Euphytica 217:45

18. Ma L, Hao CY, Liu HX, Hou J, Li T, Zhang XY (2019) Diversity and sub-functionalization of $T a G W 8$ homoeologs hold potential for genetic yield improvement in wheat. Crop J 7(6):830-844

19. Marcais G, Kingsford C (2011) A fast, lock-free approach for efficient parallel counting of occurrences of k-mers. Bioinformatics 27:764-770

20. Vurture GW, Sedlazeck FJ, Nattestad M, Underwood CJ, Fang H, Gurtowski J, Schatz MC (2017) GenomeScope: fast reference-free genome profiling from short reads. Bioinformatics 33:2202-2204

21. Luo RB, Liu BH, Xie YL, Li ZY, Huang WH, Yuan JY, He GZ, Chen YX, Pan Q, Liu YJ, Tang JB, Wu GX, Zhang H, Shi YJ, Liu Y, Yu C, Wang B, Lu Y, Han CL, Cheung DW, Yiu SM, Peng SL, Zhu XQ, Liu GM, Liao XK, Li YR, Yang HM, Wang J, Lam TW, Wang J (2015) SOAPdenovo2: an empirically improved memoryefficient short-read de novo assembler. Gigascience 1(1):18

22. Li RQ, Yu C, Li YR, Lam TW, Yiu SM, Kristiansen K, Wang J (2009) SOAP2: an improved ultrafast tool for short read alignment. Bioinformatics 25(15):1966-1967

23. Peakall R, Smouse PE (2012) GenAlEx 6.5: genetic analysis in excel. Population genetic software for teaching and research-an update. Bioinformatics 28:2537-2539

24. Perrier X, Jacquemoud-Collet JP (2006) Darwin software. Accessed from http://darwin.cirad.fr/darwin.

25. Henchion M, Hayes M, Mullen AM, Fenelon M, Tiwari B (2017) Future protein supply and demand: strategies and factors influencing a sustainable equilibrium. Foods 6:53

26. Westhoek H, Rood T, van den Berg M, Janse J, Nijdam D, Reudink M, Stehfest E (2011) The protein puzzle: the consumption and production of meat, dairy and fish in the European Union. Eur J Food Res Rev 1(3):123-144

27. Moose SP, Mumm RH (2008) Molecular plant breeding as the foundation for 21 st century crop improvement. Plant Physiol 147(3):969-977

28. Liu X, Wu SR, Xu J, Sui C, Wei JH (2017) Application of CRISPR/Cas9 in plant biology. Acta Pharm Sin B 7(3):292-302

29. Wang RK, Fan JS, Chang P, Zhu L, Zhao MR, Li LL (2019) Genome survey sequencing of Acer truncatum Bunge to identify genomic information, simple sequence repeat (SSR) markers and complete chloroplast genome. Forests 10(2):87

30. Zhang Z, Zhang JW, Yang Q, Li B, Zhou W, Wang ZZ (2021) Genome survey sequencing and genetic diversity of cultivated Akebia trifolata assessed via phenotypes and SSR markers. Mol Biol Rep 48:241-250

31. Bi QX, Zhao Y, Cui YF, Wang LB (2019) Genome survey sequencing and genetic background characterization of yellow horn based on next-generation sequencing. Mol Biol Rep 46:4303-4312

32. Ulukan H (2011) Plant genetic resources and breeding: current scenario and future prospects. Int J Agric Biol 13(3):447-454 
33. Vieira MLC, Santini L, Diniz AL, Munhoz CD (2016) Microsatellite markers: what they mean and why they are so useful. Genet Mol Biol 39(3):312-328

34. Kumar M, Choi JY, Kumari N et al (2015) Molecular breeding in Brassica for salt tolerance: importance of microsatellite (SSR) markers for molecular breeding in Brassica. Front Plant Sci 6:688
Publisher's Note Springer Nature remains neutral with regard to jurisdictional claims in published maps and institutional affiliations. 